\title{
Polymer brushes modified by photosensitive azobenzene containing polyamines
}

\author{
Alexey Kopyshev, ${ }^{1}$ Casey J. Galvin, ${ }^{2}$ Jan Genzer, ${ }^{2}$ Nino Lomadze, ${ }^{1}$ Svetlana Santer ${ }^{1 *}$ \\ ${ }^{1}$ Institute of Physics and Astronomy, University of Potsdam, 14476 Potsdam, Germany \\ ${ }^{2}$ Department of Chemical \& Biomolecular Engineering, North Carolina State University, Raleigh, \\ NC, USA
}

* TO WHOM CORRESPONDENTS SHOULD BE ADDRESSED

AUTHOR EMAIL ADDRESS: santer@uni-potsdam.de

\section{RECEIVED DATE}

TITLE RUNNING HEAD: Photosensitive brushes, photosensitive azobenzene containing polyamines

\begin{abstract}
This paper describes a strategy for preparing photosensitive polymeric grafts on flat solid surfaces by loading diblock-copolymer or homopolymer brushes with cationic azobenzene-containing surfactants. In contrast to previous work, we utilize photosensitive surfactants that bear positivelycharged polyamine head groups whose charge varies between $1+$ and $3+$. Poly(methyl methacrylateb-methacrylic acid) (PMMA-b-PMAA) brushes were prepared by employing atom transfer radical
\end{abstract}


polymerization, where the bottom poly(methyl methacrylate) block was grown first followed by the synthesis of $t$-butyl methacrylate block that after de-protection yielded poly(methacrylic acid). We used PMMA-b-PMAA brushes with constant grafting density and length of the PMMA block, and three different lengths of the PMAA block. The azobenzene-based surfactants attached only to the PMAA block. The degree of binding (i.e., the number of surfactant molecules per binding site on the brush backbone) of the surfactants to the brush depends strongly on the valence of the surfactant head-group; within the brushes the concentration of the surfactant carrying unit charge is larger than that of multivalent surfactants. We detect pronounced response of the brush topography on irradiation with UV interference pattern even at very low degree of binding (as small as 0.08) of multi-valence surfactant. Areas on the sample that receive the highest UV dose exhibit chain scission. By removing the ruptured chains from the substrate via good solvent, one uncovers a surface topographical relief grating, whose spatial arrangement follows the intensity distribution of the UV light on the sample during irradiation. Due to strong coupling of the multi-valence surfactants to the polymer brush, it was possible in some cases to completely remove the polyelectrolyte block from the PMMA layer. The application of multi-valence azobenzene surfactants for triggering brush photosensitive has important advantage over usage of surfactant with unit charge because relative to single-valence surfactants much lower concentrations of the multivalent surfactant are needed to achieve comparable response upon UV irradiation.

\section{Introduction}

Photo-reactive brushes comprising surface-tethered polymers with attached azobenzene-containing molecules exhibit strong mechanical response when irradiated with external UV light. [1-3] The azobenzene moieties are responsible for photosensitivity due to their ability to undergo an UVinduced photo-isomerization transition from a trans- to a cis-state. The photo-isomerization is 
completely reversible and can be accomplished thermally or under irradiation with light of longer wavelength. [4-6] Under photo-isomerization the molecular properties of both polymer brush and photosensitive side chains vary strongly with respect to, e.g., free volume and dipole moment to be larger for cis-isomer. Photosensitive polymer brushes can thus be thought of as forming two separate phases with different chemical potential for the azobenzene molecules or, in combination with the polymer matrix as the mechanical, responsive component, as two phases with different material properties. An external, spatially varying UV radiation field (obtained, for instance, by an interference pattern) introduces gradients in chemical potential and/or material properties. Since the azobenzene molecules are attached to the polymer matrix, opto-mechanical driving forces lead to intriguing phenomena such as the formation of surface relief gratings (SRG). [7-9] During this process, the polymer topography deforms following the distribution of the intensity or polarization of the incoming UV light. [10-19] The process takes place in air, where the polymer film is solid-like. While the formation of SRG in physisorbed polymer films has been known and well-documented for about two decades, [20-21] in polymer brush conformations the phenomenon was demonstrated only recently. $[1-3,29]$ As in the case of physisorbed film, polymer brushes deform during irradiation with UV interference pattern, yet there is a peculiarity in the process. Namely, during the SRG formation within the photosensitive polymer films, local rupturing of the polymer chains takes place in regions of high UV intensity leading to periodic topographical nano-structured corrugations of the film on the substrate, which are detected after exposing the specimens to good solvents after the UV illumination. The shape of the substrate-bound nano-pattern is determined by the morphology of the interference pattern and can feature, for instance, nano-stripes or nano-islands where the polymer chains are still attached to the solid surface. Opto-mechanically induced scission of polymer chains during the formation of surface gratings indicates that the generated stresses within the polymer films 
are large. [22] Indeed, investigating this question, we have found that metal layers placed on photosensitive polymer film can be ruptured during the SRG formation. [23, 24] Even the strongest material ever tested, i.e., graphene [25], could be stretched by the photosensitve polymer film during SRG formation applying opto-mechanical stresses of the order of $1 \mathrm{GPa}$. [26]

One can follow different strategies to render the polymer brush photosensitive. Seki at al. polymerize azobenzene modified monomers using surface-initiated ATRP to form photosensitive brushes. [27, 28] In an alternative approach one first prepares polymer brush and then the azobenzene molecules are attached as side chains either covalently [1, 2] or ionically. [3, 29] In the latter case the requirement is that the brush and azobenzene molecules possess opposite charges. We have followed this strategy to functionalize brushes consisting of poly(methylmethacrylate-bmethacrylic acid) (PMMA-b-PMAA) diblock copolymers [3] and PMAA homopolymer [29] with cationic azobenzene-containing surfactants that attached selectively to the charged PMAA chains. We demonstrated that in the case of diblock-copolymer brushes rupture occurred at the link connecting PMMA (non-functionalized) and PMAA (photosensitive). [3] Depending on the irradiation intensity, up to nearly $90 \%$ of the PMAA chains can be ruptured during irradiation and subsequently removed locally from the intact PMMA block during treatment with good solvent, leaving behind a nano-structured brush consisting of lines or small islands. In reference 29 we explored the behavior of systems featuring three surfactants that possessed the same charged headgroup and differed in the length of the hydrophobic spacer connecting the head-group and the azobenzene unit. We demonstrated that surfactants of larger hydrophobic species did not penetrate deep into the brush. Since rupturing of polymer chains occurred predominantly in the regions of high surfactant density, a leftover layer of PMAA non- functionalized with surfactants remained on the substrate. 
In this work we show that complete local removal of polymer chains during the formation of SRG is achieved even at very low concentration of surfactant within the brush. In reference 3 , the binding degree of the azobenzene containing surfactant was $\beta=0.8$ (i.e., 0.8 surfactant molecules per one carboxyl group within the brush). In this paper we show that even a 10 fold lower degree of binding, i.e., $\beta=0.08$, is sufficient to induce a comparable degree of polymer chain scission.

To achieve this we load PMMA-b-PMAA diblock-copolymer brushes with azobenzenecontaining surfactants consisting of polyamines as head-groups and a hydrophobic tail, in which the azobenzene is incorporated. Mimicking natural polyamines (spermidine (3+) and spermine (4+)) [3033] one utilizes synthetic polyamines for compaction of DNA [34-38] and, when functionalized with azobenzene, for light driven remote control of the size of DNA and microgels. [39] We chose the polyamine head-group because of its low toxicity. $[37,38]$ The charge at the head group is varied between $1+$ and $3+$, depending on the $\mathrm{pH}$ and the number of amine groups. The degree of binding of the surfactant to the brush depends strongly on the number of amino groups in the surfactant head. For instance, for the same brush the degree of binding, i.e., the number of surfactant molecules per binding site on the brush backbone, decreases from 0.24 for a polyamine with $1+$ charge to 0.08 for a polyamine with $3+$ charges. Interestingly, upon UV irradiation, the extent of polymer chain scission of brushes loaded with multivalent surfactants even at such lower degrees of binding is comparable with systems featuring surfactants bearing $1+$ head-groups. We attribute this behavior to stronger coupling of the photo-sensitive molecules actuator to the polymer matrix and thus more effective transduction of the opto-mechanical forces. 


\section{Experimental Section}

Synthesis of PMMA-PMAA polymer brush.

PMMA-b-PMAA diblock-copolymer brushes were synthesized as described elsewhere. [3] In short, first PMMA brushes were synthesized from ATRP initiator (11-(2-Bromo-2-methyl)propionyloxy undecyltrichlorosilane, BMPUS) deposited on a silicon wafer or quartz glass following a previously reported method. [40,41] In the second step PtBMA blocks were grown from the PMMA chains to form PMMA-b-PtBMA diblock-copolymer brushes. [42] Samples were removed from the polymerization solution at certain time intervals to achieve different length blocks of PtBMA. In the final step, the PMMA-b-PtBMA grafts were converted to PMMA-b-PMAA diblock-copolymer brushes by exposing them to a $50 \%(\mathrm{v} / \mathrm{v})$ of trifluoroacetic acid in dichloromethane. Based on evolution of the brush thickness, the reaction appeared complete within thirty minutes of exposure time.

Synthesis of photosensitive azobenzene containing polyamines.

The cationic azobenzene containing polyamines were synthesized as described elsewhere. [38] At first azo-coupling of p-buthylaniline with phenol and following subsequent reaction with 1,6dibromoalkane was carried out. The next step involved reactions with ethylenediamine, diethylenetriamine and tris(2-aminoethyl)amine. The azobenzene containing polyamines differ in the number of amine group in the head; i.e., 1, 2 and 3 (Figures $\mathbf{1} \mathbf{a 1 ,} \mathbf{a} 2, \mathbf{a 3}$, respectively). In the following the polyamines are termed as Azo-En, Azo-Deta and Azo-Tren. The polyamines were dissolved in water (MilliQ) and kept in the dark for several days to ensure complete relaxation to the trans configuration. Depending on the $\mathrm{pH}$ value, the charge varies from $1+$ to $3+$ increasing from Azo-En to Azo-Deta and to Azo-Tren. The experiments were conducted at $\mathrm{pH}=5$ at which the 
amino groups are partially protonated. [38] The fourth photosensitive surfactant discussed in the paper is azobenzene-containing trimethylammonium bromide (Azo-TMAB) (Figure 1 a4), described in previous publication. [43]

The photo-isomerization behavior of the polyamines.

All three polyamine compounds and Azo-TMAB have similar structure of a hydrophobic tail, in which the azobenzene moiety is integrated (Figure 1). The azobenzene group with a short alkyl chain $\left(\mathrm{CH}_{2}\right)_{3}-\mathrm{CH}_{3}$ at a para position is connected to the polyamine part through a hydrophobic spacer bearing $6 \mathrm{CH}_{2}$ groups. The photoisomerization of polyamine was found to be similar to previously described cationic surfactant Azo-TMAB. [44] The trans isomer of all three polyamines and AzoTMAB displayed a characteristic absorption band $\left(\pi-\pi^{*}\right.$ transition of the azobenzene unit in the trans conformation) with a maximum at $351 \mathrm{~nm}$. The UV/vis spectrum of the cis isomer features two absorption bands with maxima at $313 \mathrm{~nm}\left(\pi-\pi^{*}\right.$ transition) and at $437 \mathrm{~nm}$ (n- $\pi^{*}$ transition). The lifetime of the cis isomer in the brush is $\sim 22$ hours; therefore, the spectra do not change considerably after the irradiation is turned off. [18] Irradiation at $325 \mathrm{~nm}$ results in an intermediate ratio of the trans and cis isomers, defined by the ratios of the extinction coefficients at the corresponding wavelengths. The band with maximum at $\sim 240 \mathrm{~nm}$ corresponds to the absorption of the $\pi$-conjugated benzene rings present in both isomers.

\section{Binding of photosensitive surfactants.}

The PMMA-b-PMAA brushes were placed in polyamine solution of a given concentration for 1 hour at room temperature followed by drying under nitrogen flow. The interactions between the surfactants and the brush are driven by the interplay among hydrophobic, ionic and H-bond forces. 
Any unbound molecules were removed by rinsing the sample in a water bath. The thickness of a sample increased due to absorption of the surfactant. The degree of binding $(\beta)$ was determined by the following equation: [45]

$$
\beta=\frac{\frac{h_{b}^{a z o}}{h_{b}}-1}{\frac{M_{0}^{a z o+M A A}}{M_{0}^{M A A}}-1}
$$

In Equation (1), $h_{b}$ and $h_{b}{ }^{a z o}$ are the brush dry thicknesses (only the thickness of the PMAA block) before and after complex formation, respectively, $M_{0}{ }^{M A A}(=86 \mathrm{Da})$ are molecular weights of the repeat units before complexation and $M_{0}{ }^{a z o+M A A}=481,520,563 \mathrm{Da}$ are molecular weights of the repeat units after complexation with polyamines for Azo-En, Azo-Deta, Azo-Tren, respectively.

\section{Methods}

Atomic force microscopy (AFM) (Nanoscope V, Veeco, USA) was employed to characterize surface topography of PMMA-PMAA brushes. The microscope was operated in tapping mode, using commercial tips (NanoWorld) with a resonance frequency of $\sim 320 \mathrm{kHz}$, and a spring constant of $\sim 40$ $\mathrm{N} / \mathrm{m}$. The experiments were carried out in air, at room temperature of $23^{\circ} \mathrm{C}$, and constant humidity of $55 \%$, in a room with yellow light to avoid any premature isomerization of the surfactant.

UV-visible spectra were measured by a Cary 5000 UV-Vis-NIR spectrophotometer (Varian, Inc.).

The Lloyd's mirror scheme with He-Cd laser (Kimmon) operating at $\lambda=325 \mathrm{~nm}$ (total power of $\sim 3 \mathrm{~mW}$ ) was used as UV interference lithography. The periodicity of the interference pattern is given by $\mathrm{d}=\lambda / 2 \sin \theta$, where $\lambda$ is the wavelength of the incident light, and $\theta$ is the angle between the incoming laser beam and the mirror surface. Irradiation time of 15 minutes and the periodicity of interference pattern of $1.5 \mu \mathrm{m}$ were kept constant for all experiments. 


\section{Results and Discussion}

In this study we have investigated three diblock-copolymer PMMA-b-PMAA brushes with the same grafting density and molecular weight of the bottom PMMA block, different molecular weight of the dangling PMAA block. Specifically, three molecular weights of the PMMA block were examined: 32, 84 and $111 \mathrm{kDa}$ for brushes I, II, and III, respectively (Table 1). The brushes can be viewed as bi-layer films consisting of PMMA and PMAA blocks (Figure 1b).
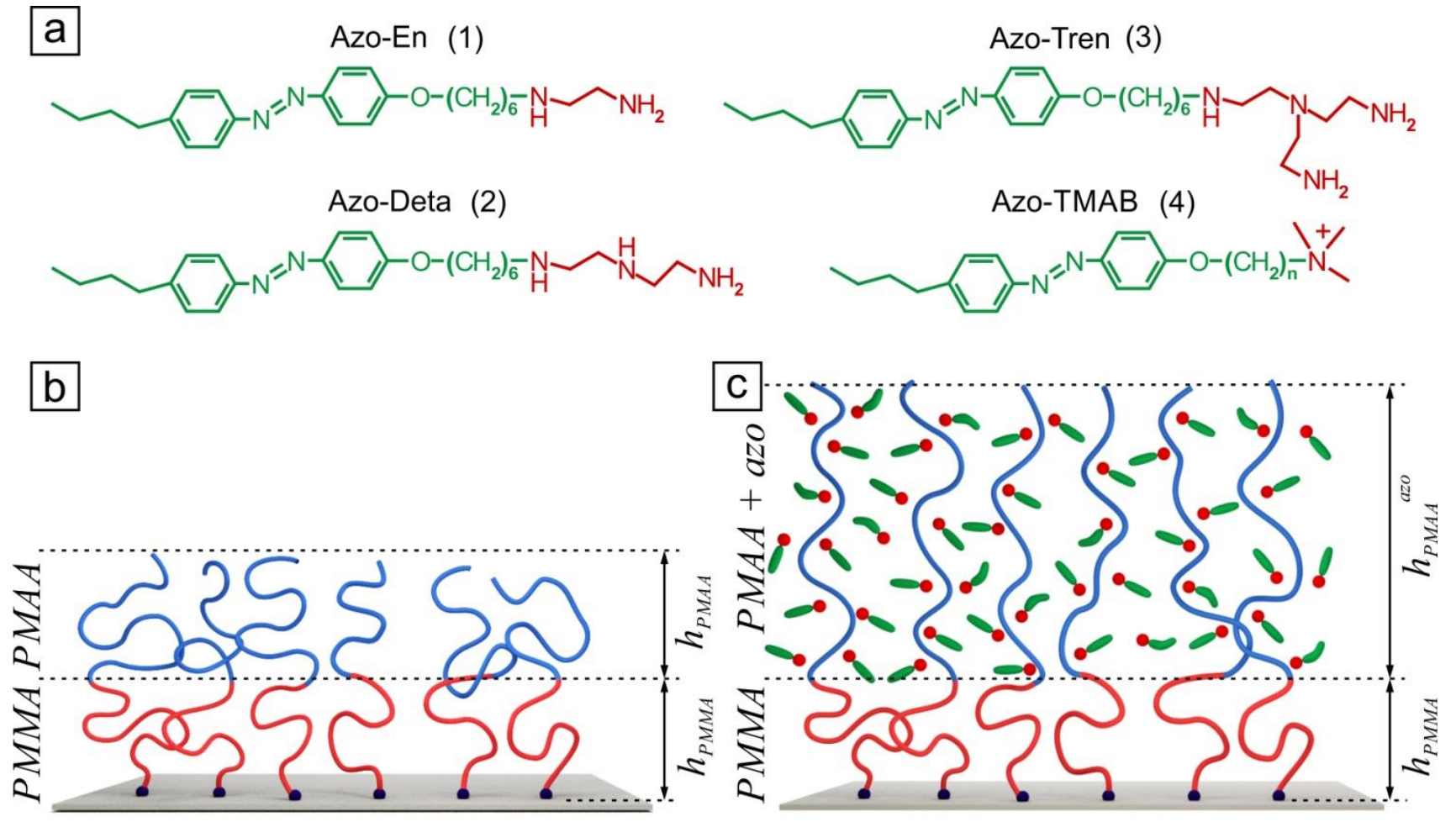

Figure 1. (a) Chemical structures of the studied photosensitive molecules. The molecules differ in the nature of the charged head group: (1) Azo-En, (2) Azo-Deta, (3) Azo-Tren, (4) Azo-TMAB. (b) Scheme of the diblock-copolymer brush (red block is PMMA, blue block is PMAA). (c) Scheme of the brush loaded with photosensitive molecules interacting only with PMAA block.

The height of the first PMMA block attached to a solid surface is $20 \mathrm{~nm}$ for all brushes, while the height of the second PMAA block varies from 19 to 50 and to $66 \mathrm{~nm}$ for brushes I, II and III, respectively (Table 1). Figure 1a shows chemical structures of the studied photosensitive 
polyamines (1: Azo-En, 2: Azo Deta, and 3: Azo-Tren) and the chemical structure of the cationic azobenzene containing surfactant (Azo-TMAB) reported elsewhere $[3,18]$ and included here for comparison.

To load brush with photosensitive molecules, the film was exposed to surfactant solution (concentration $1 \mathrm{mM}$ ) for 1 hour followed by rinsing with water and drying. The polyamines interact ionically, hydrophobically or through H-bonding with the brush resulting in the total height increase as shown in Figure 1c. We have found that the molecules adsorb only into the PMAA block by performing a control experiment. Here the PMMA brush was exposed to surfactant solution and post treated as described above for the case of diblock copolymer brush. No change in the thickness of the PMMA brush was observed, indicating the absence of polyamines within the brush. The same behavior was found for azobenzene containing surfactant as well. [3]

Table 1 lists the change in the height of the PMAA block and the degree of surfactant binding for the two polyamines Azo-En and Azo-Tren. In the following we will also discuss the interaction of AzoDeta and Azo-TMAB with brushes (Supporting Information Figure S1).

\begin{tabular}{|c|c|c|c|c|c|c|c|c|c|c|}
\hline \multirow[b]{2}{*}{ brush } & \multirow[b]{2}{*}{$\begin{array}{c}\mathbf{h}_{\text {PMMA }}, \\
\mathbf{n m}\end{array}$} & \multirow[b]{2}{*}{$\begin{array}{c}\mathbf{h}_{\text {PMAA }}, \\
\text { nm }\end{array}$} & \multirow[b]{2}{*}{$\begin{array}{c}M_{n}, \\
10^{4}, \mathrm{~g} / \mathrm{mol}\end{array}$} & \multirow[b]{2}{*}{$\Sigma$} & \multicolumn{3}{|c|}{ Azo-En } & \multicolumn{3}{|c|}{ Azo-Tren } \\
\hline & & & & & $\begin{array}{c}\mathbf{h}_{\text {PMAA+azo, }} \\
\mathbf{n m}\end{array}$ & $\beta$ & $\begin{array}{c}\mathbf{h}_{\text {lines' }}, \\
\mathbf{n m}\end{array}$ & $\begin{array}{c}\mathbf{h}_{\text {PMAA+azo }} \\
\mathbf{n m}\end{array}$ & $\beta$ & $\begin{array}{c}\mathbf{h}_{\text {lines }}, \\
\mathbf{n m}\end{array}$ \\
\hline I & \multirow{3}{*}{$20 \pm 0.5$} & $19 \pm 0.5$ & 3.2 & 1.8 & $55 \pm 5$ & 0.41 & $19 \pm 4$ & $37 \pm 5$ & 0.17 & $19 \pm 3$ \\
\hline II & & $50 \pm 0.5$ & 8.4 & 4.7 & $121 \pm 5$ & 0.31 & $60 \pm 5$ & $85 \pm 10$ & 0.13 & $80 \pm 2$ \\
\hline III & & $66 \pm 0.5$ & 11.1 & 6.2 & $140 \pm 5$ & 0.24 & $80 \pm 5$ & $96 \pm 5$ & 0.08 & $90 \pm 2$ \\
\hline
\end{tabular}

Table 1. Molecular parameter of the PMMA-PMAA diblock-copolymer brushes. The grafting density, $\sigma=0.4 \mathrm{~nm}^{-2}$, is determined as described elsewhere. [46] The number average molecular weight, $\mathrm{M}_{\mathrm{n}}$, is calculated according to the known height of the brush: $M_{n}=\frac{h \cdot \rho \cdot N_{A}}{\sigma}$, where $\rho$ is the density of PMAA $1.12 \mathrm{~g} / \mathrm{cm}^{3} . \mathrm{h}_{\text {PMAA }}{ }^{\text {azo }}=\left(\mathrm{h}_{\text {total }}{ }^{\text {azo }}\right.$ - $\left.\mathrm{h}_{\text {PMMA }}\right)$ is the height of the PMAA block after loading the brush with azobenzene surfactants. $\beta$ is the degree of surfactant binding. $h_{\text {lines }}$ is the 
height of the SRG after irradiation and subsequent treatment with DMF. $\Sigma$ is the reduced grafting density (for more details see the text below).

Figure 2 shows the change in the height of the PMAA block after loading with azobenzene containing surfactants (Azo-En Figure 2a, Azo-Tren Figure 2b) depending on the brush type.
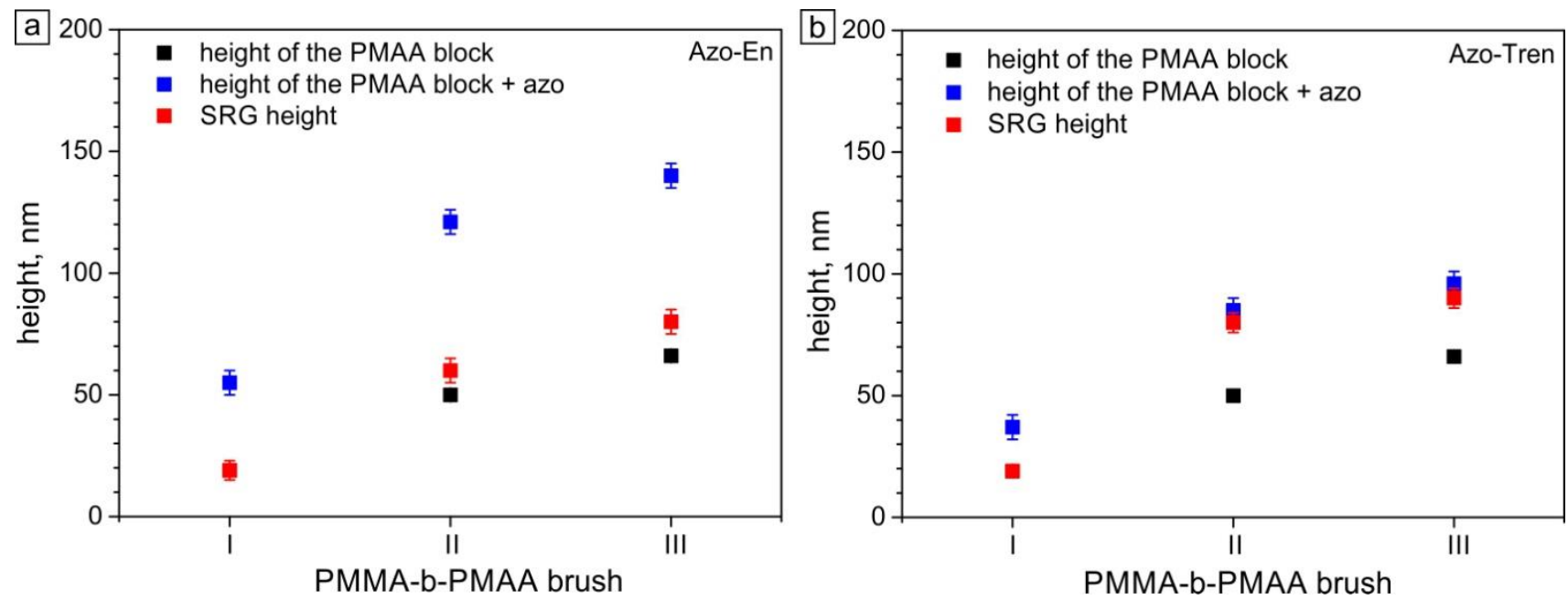

Figure 2. Dependence of the height of the PMAA block on the brush type before loading with the surfactants (black squares), after complexation with the surfactants (blue squares): (a) Azo-En, (b) Azo-Tren. The red squares indicate the SRG height after irradiation with UV interference pattern and subsequent treatment with a good solvent.

One can calculate the degree of binding, $\beta$, by measuring the height of the brush using AFM scratch experiment before and after loading with photosensitive molecules (Table 1). As can be inferred from Figure 2 and Table 1, $\beta$ decreases with increasing molecular weight of the PMAA block for both surfactants. When comparing the surfactants, one also sees that Azo-Tren with the 3 charges at the head group binds less than the surfactant with 1.5 charges (Azo-En). In the case of Azo-En molecules the degree of binding is the largest among all polyamines and reaches 0.41 for the brush I (Table 1, Figure 3), indicating that $41 \%$ of all carboxyl groups bind with the polyamine. For the same surfactant $\beta$ increases with decreasing molecular weight of the PMAA block (Table 1). This can be understood by considering a reduced grafting density parameter, $\Sigma$, which is defined as 
$\Sigma=\sigma \cdot \pi \cdot R_{g}^{2}$, where $R_{g}$ is radius of gyration of a tethered chain at specific experimental conditions of solvent and temperature. $\Sigma$ estimates the degree of crowding of the brush, since it shows the number of tethered chains occupying an area defined by a free polymer chain of the same molecular weight. [47] As it can be seen from the Table 1, the reduced grafting density increases with molecular weight from $\Sigma=1.8$ for the brush I to $\Sigma=6.2$ for the brush III. Thus, brush III is more crowded and can absorb less surfactant.

We chose brush III with the highest molecular weight of the PMAA block to compare the degree of binding among different surfactants. Figure 3 shows the degree of binding depending on the surfactant type. In the Supporting Information Figure S1, one can find the molecular parameters of the brush III loaded with the Azo-Deta and Azo-TMAB.

The number of surfactant molecules bound in the brush decreases with increasing the number of charges at the head group. The Azo-TMAB surfactant, shown for comparison, has the largest number of the incorporated molecules, $\beta=0.9$. The polyamines with 2 (Azo-Deta) and 3 (Azo-Tren) charges exhibit similarly low degree of binding $(0.07 \pm 0.01)$ for brush III (Figure 3). This difference can be explained by the following arguments. The uptake and coupling of photosensitive molecules into the brush takes place as a result of an ion exchange reaction between brush counterions $\left(\mathrm{H}^{+}\right)$and cationic surfactants. The surfactant carrying unit charge on the head group is able to substitute only one brush counterion, while a multivalent surfactant ensures release of larger number of brush counterions. 


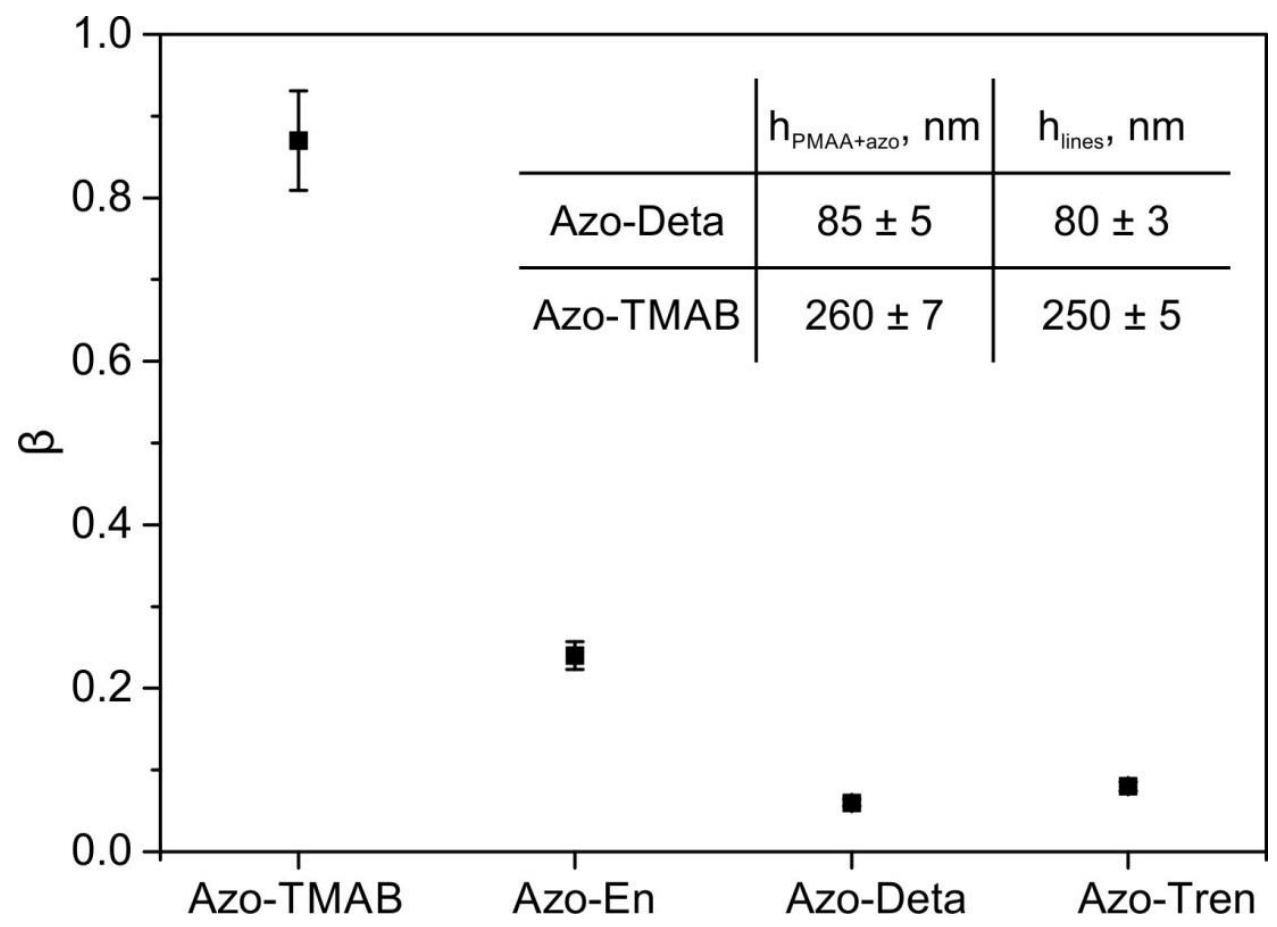

Figure 3. Dependence of the degree of binding of four photosensitive molecules on the nature of charged head group. The short names of the surfactants are explained in the Figure 1a.

In this case the ion exchange is more favored due to a larger entropy gain associated with escaping counterions from the brush. It means that at the same concentration of the surfactants with one and three charges the brush exposed to these surfactant solutions will collapse to a large degree in the case of multivalent surfactants, since it results in more pronounced drop of osmotic pressure inside the brush. This, in turn, results in a smaller concentration of the multivalent surfactants within the brush, and thus, smaller degree of binding. As will be shown subsequently, even a low concentration of the photosensitive surfactants loaded within the brush is sufficient to render the graft highly responsive to UV irradiation.

To obtain nano-scaled stripes of PMAA chains on top of PMMA blocks (Figure 4), we first irradiate the photosensitive brushes with UV interference pattern $(\lambda=325 \mathrm{~nm}, \mathrm{D}=1.5 \mu \mathrm{m})$ followed by subsequent exposure to DMF, a good solvent for both PMAA and the surfactants. As it was recently 
reported, [3,18] there is a formation of surface relief grating (SRG) within the brush under irradiation.
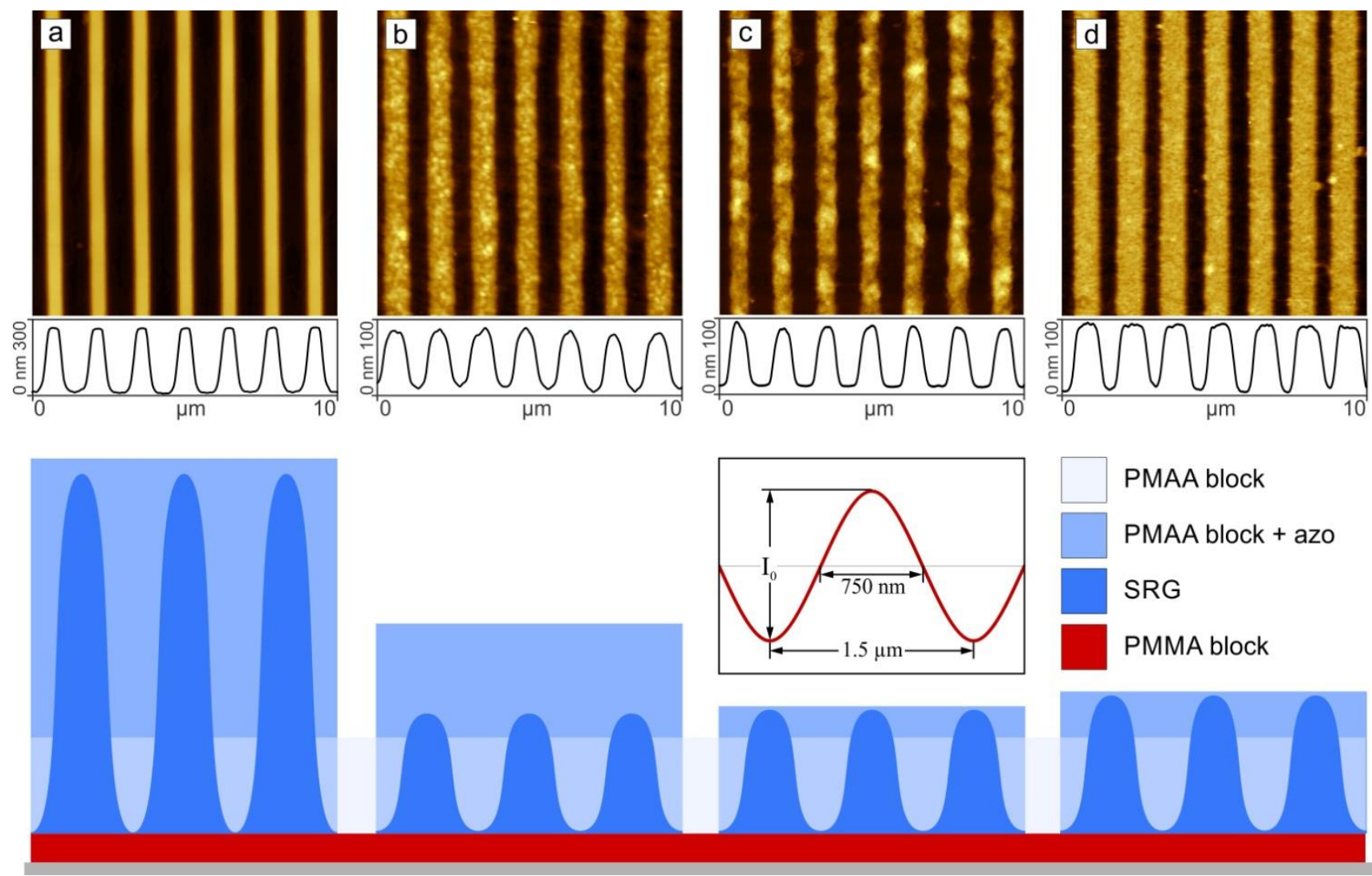

Figure 4. AFM micrographs of the structured surfaces of the PMMA-PMAA photosensitive brushes and corresponding cross-sectional scheme (below) for brush III loaded with different surfactants: (a) Azo-TMAB, (b) Azo-En, (c) Azo-Deta, (d) Azo-Tren. Light blue color shows the PMAA block before functionalization, red color depicts the PMMA block. After functionalization with the photosensitive surfactants the thickness of the PMAA block (blue color) increases. After irradiation with interference pattern and subsequent treatment with DMF solvent, the formation of the parallel ordered stripes (dark blue color) consisting of PMAA chains are observed. The insert shows a sinusoidal profile of the UV interference pattern.

The height of the $\mathrm{SRG}$ is $\sim 10 \mathrm{~nm}$ (just after irradiation) for all surfactants. However, during irradiation the opto-mechanical stresses are strong enough to induce local scission of the polymer chains from of the areas on the specimen that received the highest UV dosage. The scission of PMAA chains appears at the PMMA-b-PMAA interface as can be seen from the cross-sectional 
scheme in Figure 4 and Figure 2. Moreover, in the case of the Azo-En surfactant (with 1.5 charge at the head), the polyelectrolyte chains rupture even at the areas of minimum intensity resulting in the height of the SRG that is smaller than the thickness of the functionalized PMAA block. The roughness of the brush surface before and after irradiation is larger in the case of polyamine surfactants. Thus, the brush loaded with Azo-TMAB has $1.4 \mathrm{~nm}$ root-mean square (rms) roughness (measured over $10 \times 10 \mu \mathrm{m}^{2}$ area), while the brushes complexed with polyamines has a rms of $\sim 4$ $\mathrm{nm}$. In addition, the profile of the SRG differs for Azo-TMAB and polyamine surfactants (Figure 4). In the case of Azo-TMAB modified brush, the profile appears to be sinusoidal, matching the intensity distribution of the UV interference pattern. In contrast, in the case of polyamine modified brushes, the profile resembles channels with a flat top (cf. Figure 4d). This behavior may be related to the strength of the interactions between the surfactants and the polymer brush. Indeed, the irradiation field acts only on the photo-responsive azobenzenes, that undergoes photo-isomerization from the stable trans- to a meta stable cis-conformation with corresponding changes in dipole moment, volume, and orientation. These opto-chemical forces are transduced to mechanical response within azobenzene-containing polymer films. The coupling between the active molecular actuators and the "passive" polymer matrix is achieved via hydrophobic, H-bonding and ionic interactions between the surfactants and the polymer chains. Since polyamine surfactants bear larger number of charges, they should be coupled more strongly to the polymer and thus be more effective in pulling out of the polymer chains. Indeed, as can be seen from Figure 4 only a small amount of the polyamine surfactants is needed to remove completely the PMAA block from the UV irradiated areas, while in the case of Azo-TMAB molecules with a comparable degree of binding $\sim 30 \%$ of the total brush thickness is left behind on the same areas of the specimen. [18] Although the coupling between the polyamines and the polymer chains is strong, the concentration of the photosensitive 
units within the brush is low; this results in brush scission only in the regions of maximal UV intensity. The distribution of the intensity within the interference pattern is sinusoidal, i.e., at the period of $1.5 \mu \mathrm{m}$, the intensity drops two-fold within the $750 \mathrm{~nm}$ near the maxima (see insert in Figure 4). The width on the channel like grating in the case of Azo-Deta and Azo-Tren (Figure 4c and Figure 4d) is $~ 750 \mathrm{~nm}$.
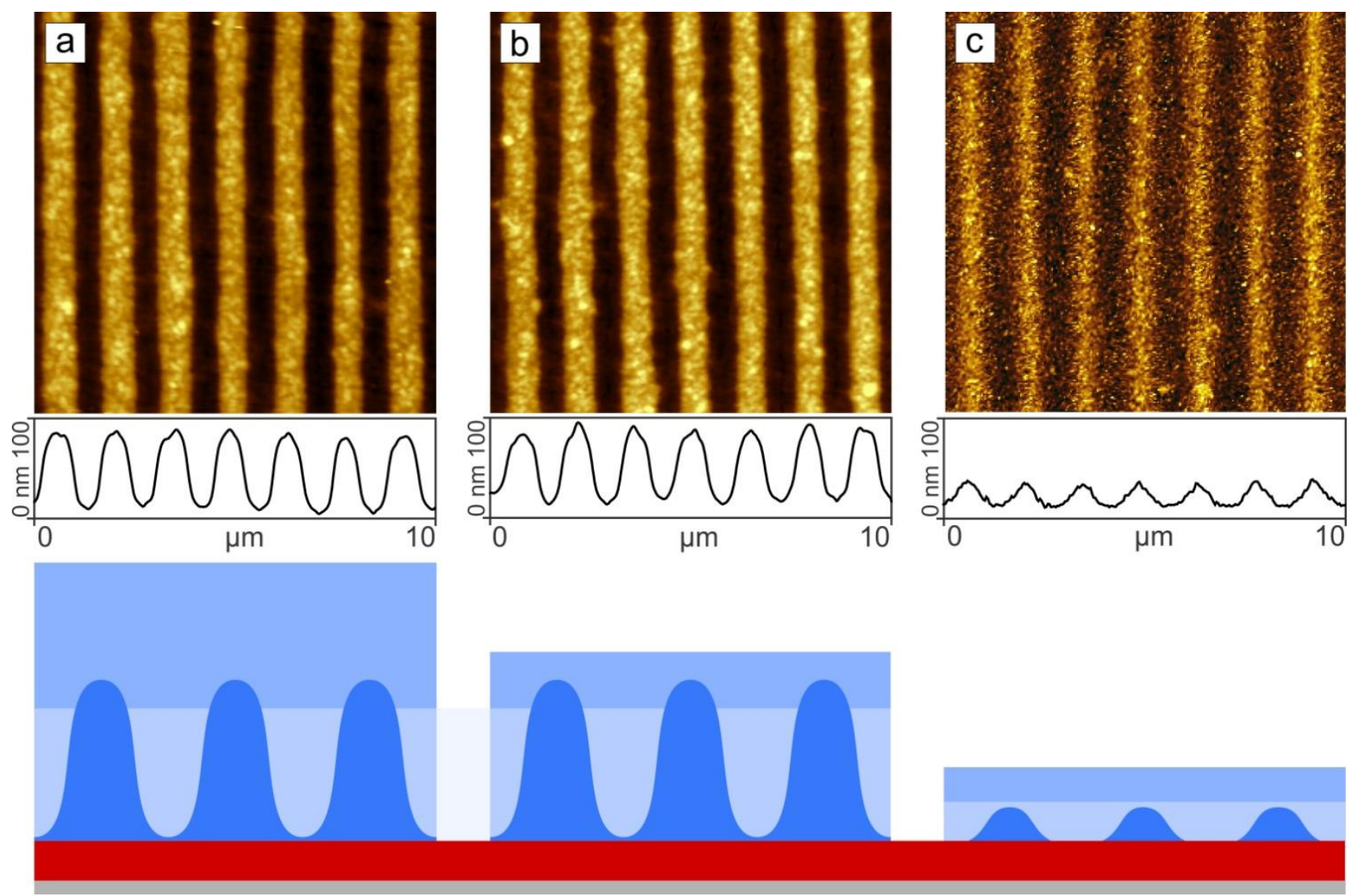

Figure 5. AFM micrographs of the SRG formed within the brush III loaded with Azo-En at different degree of binding: brush III (a) $\beta=0.24$, (b) $\beta=0.09$; (c) brush I, $\beta=0.41$.

In the case of Azo-En the strong coupling to the brush results even in the partial removal of the polymer chains from the areas of minimal intensity (Figure $4 \mathbf{b}$ and Figure 5a). Here the height of the SRG is smaller than the thickness of the PMAA block loaded with surfactants (see scheme in Figure 4b and Figure 5a). However, when the degree of binding decreases to 0.09, scission of 
polymer chains is observed predominantly at the topography minima (Figure 5b). Before irradiation the height of the PMAA block coupled to Azo-En is $\mathrm{h}_{\mathrm{PMAA}+\mathrm{zzo}}=90 \pm 5 \mathrm{~nm}$, while at the height of the SRG is $\mathrm{h}_{\text {lines }}=80 \pm 5 \mathrm{~nm}$.

Strong coupling between the photo-active actuators and the polymer chains results in some cases in complete removal of the PMAA block during irradiation with UV interference pattern followed by subsequent exposure to DMF. For instance, in the case of brush I loaded with Azo-Deta and AzoTren $(\beta=0.17)$, there is only PMMA block left (the AFM micrograph is not shown here since it is just a flat surface). The brush I/Azo-En $(\beta=0.41)$ complex responds strongly to irradiation, so that only $15 \mathrm{~nm}$ thick gratings are left after irradiation (Figure 5c), at the initial thickness of the PMAA /Azo-En block of 55nm.

In our recent publication, we have reported that Azo-TMAB can be removed from the brush by long extraction in the water bath. [18] In the case of the polyamines, the significant removal of the surfactants was not observed after 24 hours of water extraction.

\section{Conclusions}

We present investigation of the formation of macromolecular complexes featuring PMMA-b-PMAA diblock copolymer brushes and photosensitive cationic surfactants and study their response to UV radiation. The brushes were prepared using ATRP in such a way, that the molecular weight of the first block attached to a solid surface (PMMA) and the grafting density of the brushes were kept constant, while the molecular weight of the dangling block (PMAA) was varied from 32 to $111 \mathrm{kDa}$. The surfactant molecules feature positively charged head group consisting of polyamines to which the azobenzene group is attached to through the hydrophobic spacer. Depending on the number of amine groups, the valence of the head group differs between 1 and 3. For comparison, we present 
also the results on Azo-TMAB surfactant where the head group bears a permanent single positive charge. When the brushes are exposed to a surfactant solution, the surfactants are adsorbed within the PMAA block and its thickness increases. We determined the degree of binding $(\beta)$ by analyzing the increase in the thickness of the brush after surfactant coupling. For the same surfactant $\beta$ increases with decreasing the molecular weight of the PMAA block. Additionally, within one chosen brush, $\beta$ depends on the number of charges at the head group; $\beta$ is smaller for surfactant having a higher valence at the head-group. The smallest degree of binding $(\beta=0.07)$ was obtained for the brush with the largest molecular weight of the PMAA block loaded with the surfactant Azo-Tren bearing 3 charged at the head group. $\beta=0.07$ indicates that only $7 \%$ of the carboxylate groups within the brush interact with the surfactant. In response to irradiation featuring an UV interference pattern, polymer brushes loaded with photosensitive surfactants form surface relief gratings (SRG) of topography that is associated with local rupturing of PMAA chains on the same that received the highest UV dose. The extent of polymer chain scission is comparable for surfactants of different valence, despite of the small equilibrium concentrations of the multi-valence surfactants in the brush. We explain this phenomenon by considering stronger interaction of multi-valent surfactant with the brush relative to univalent surfactants. The presence of multi-valent azobenzene surfactants allows for more effective transduction of the opto-mechanical forces from the photo actuator azobenzene molecules to the polymer matrix.

ACKNOWLEDGMENTS: This research is supported by the DFG Priority Programme "Microswimmers - From Single Particle Motion to Collective Behaviour" (SPP 1726) and VW Stiftung (Germany). JG acknowledges financial support from the National Science Foundation under Grant No. DMR-1404639. 

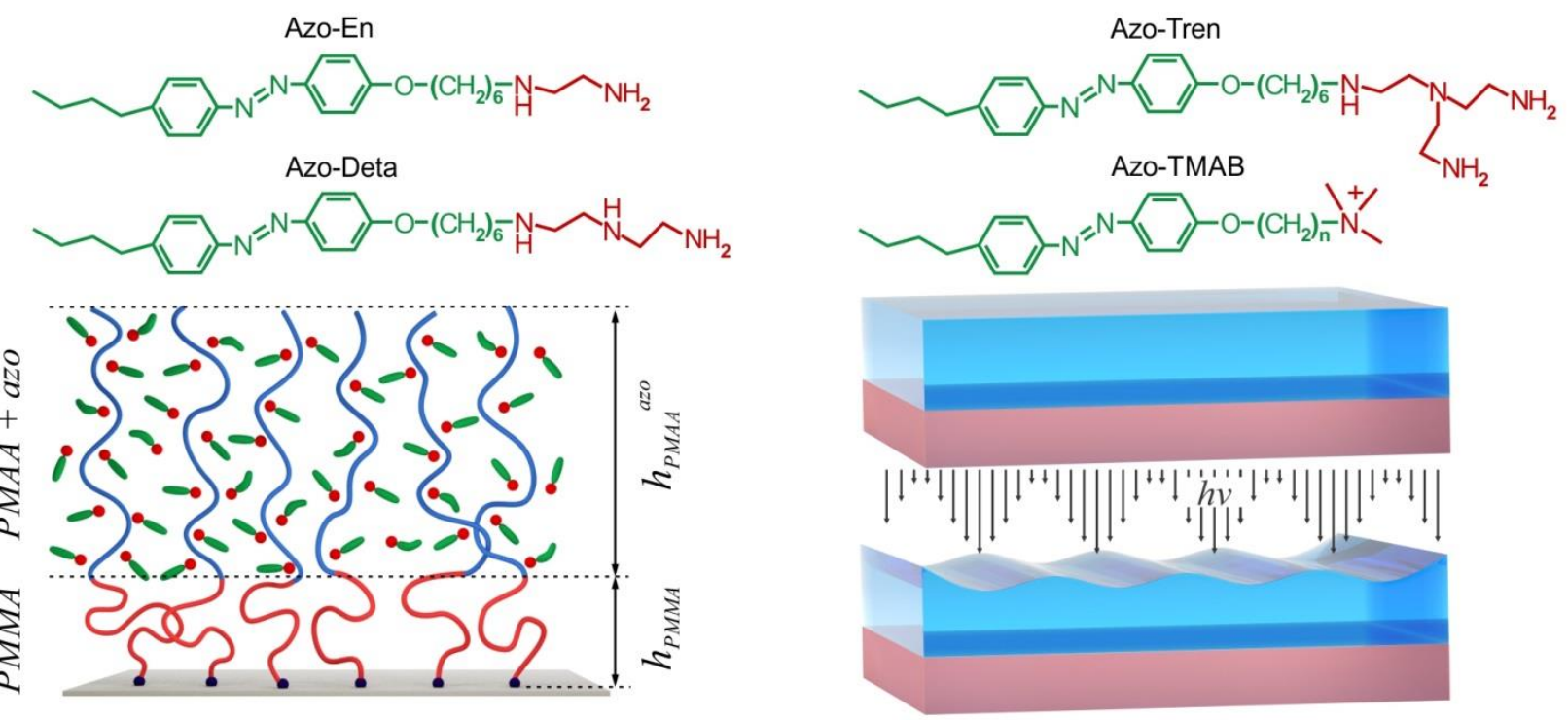


\section{References}

[1] Lomadze N, Kopyshev A, Ru $\square$ he J, Santer S. Light-Induced Chain Scission in Photosensitive Polymer Brushes. Macromolecules 2011;44:7372-7. doi:10.1021/ma201016q.

[2] Schuh C, Lomadze N, Rühe J, Kopyshev A, Santer S. Photomechanical degrafting of azofunctionalized poly(methacrylic acid) (PMAA) brushes. J Phys Chem B 2011;115:10431-8. doi:10.1021/jp2041229.

[3] Kopyshev A, Galvin CJ, Genzer J, Lomadze N, Santer S. Opto-mechanical scission of polymer chains in photosensitive diblock-copolymer brushes. Langmuir 2013;29:13967-74. doi:10.1021/la403241t.

[4] Rau H. Photoisomerization of azobenzenes. In: Rabek J, editor. Photochem. Photophysics, Boca Raton, FL: CRC Press; 1990, p. 119-42.

[5] Bandara HMD, Burdette SC. Photoisomerization in different classes of azobenzene. Chem Soc Rev 2012;41:1809-25. doi:10.1039/c1cs15179g.

[6] Zhao Y, Ikeda T, editors. Smart Light-Responsive Materials: Azobenzene-Containing Polymers and Liquid Crystals. Hoboken, NJ, USA: John Wiley \& Sons, Inc.; 2009. doi:10.1002/9780470439098.

[7] Mechau N, Saphiannikova M, Neher D. Molecular tracer diffusion in thin azobenzene polymer layers. Appl Phys Lett 2006;89:251902. doi:10.1063/1.2405853.

[8] Mechau N, Saphiannikova M, Neher D. Dielectric and Mechanical Properties of Azobenzene Polymer Layers under Visible and Ultraviolet Irradiation. Macromolecules 2005;38:3894902. doi:10.1021/ma0479316.

[9] Emoto A, Uchida E, Fukuda T. Optical and Physical Applications of Photocontrollable Materials: Azobenzene-Containing and Liquid Crystalline Polymers. Polymers (Basel) 2012;4:150-86. doi:10.3390/polym4010150.

[10] Goldenberg LM, Kulikovsky L, Kulikovska O, Tomczyk J, Stumpe J. Thin layers of low molecular azobenzene materials with effective light-induced mass transport. Langmuir 2010;26:2214-7. doi:10.1021/la9040562.

[11] Goldenberg LM, Gritsai Y, Stumpe J. Efficient surface relief grating generated in azobenzene-containing material using an He-Ne laser. J Opt 2011;13:075601. doi:10.1088/2040-8978/13/7/075601.

[12] Kulikovska O, Gharagozloo-Hubmann K, Stumpe J, Huey BD, Bliznyuk VN. Formation of surface relief grating in polymers with pendant azobenzene chromophores as studied by AFM/UFM. Nanotechnology 2012;23:485309. doi:10.1088/0957-4484/23/48/485309.

[13] Toshchevikov V, Saphiannikova M, Heinrich G. Light-induced deformation of azobenzene elastomers: a regular cubic network model. J Phys Chem B 2012;116:913-24. doi:10.1021/jp206323h.

[14] Toshchevikov V, Saphiannikova M, Heinrich G. Microscopic theory of light-induced deformation in amorphous side-chain azobenzene polymers. J Phys Chem B 2009;113:503245. doi:10.1021/jp8078265.

[15] Saphiannikova M, Geue TM, Henneberg O, Morawetz K, Pietsch U. Linear viscoelastic analysis of formation and relaxation of azobenzene polymer gratings. J Chem Phys 2004;120:4039-45. doi:10.1063/1.1642606. 
[16] Saphiannikova M, Neher D. Thermodynamic theory of light-induced material transport in amorphous azobenzene polymer films. J Phys Chem B 2005;109:19428-36. doi:10.1021/jp053249h.

[17] Yadavalli NS, Saphiannikova M, Santer S. Photosensitive response of azobenzene containing films towards pure intensity or polarization interference patterns. Appl Phys Lett 2014;105:051601. doi:10.1063/1.4891615.

[18] Yadavalli NS, Santer S. In-situ atomic force microscopy study of the mechanism of surface relief grating formation in photosensitive polymer films. J Appl Phys 2013;113:224304. doi:10.1063/1.4809640.

[19] Priimagi A, Shimamura A, Kondo M, Hiraoka T, Kubo S, Mamiya J-I, et al. Location of the Azobenzene Moieties within the Cross-Linked Liquid-Crystalline Polymers Can Dictate the Direction of Photoinduced Bending. ACS Macro Lett 2012;1:96-9. doi:10.1021/mz200056w.

[20] Rochon P, Batalla E, Natansohn A. Optically induced surface gratings on azoaromatic polymer films. Appl Phys Lett 1995;66:136-8. doi:10.1063/1.113541.

[21] Kim DY, Tripathy SK, Li L, Kumar J. Laser-induced holographic surface relief gratings on nonlinear optical polymer films. Appl Phys Lett 1995;66:1166-8. doi:10.1063/1.113845.

[22] Singleton TA, Ramsay KS, Barsan MM, Butler IS, Barrett CJ. Azobenzene photoisomerization under high external pressures: testing the strength of a light-activated molecular muscle. J Phys Chem B 2012;116:9860-5. doi:10.1021/jp3060872.

[23] Yadavalli NS, Korolkov D, Moulin J-F, Krutyeva M, Santer S. Probing opto-mechanical stresses within azobenzene-containing photosensitive polymer films by a thin metal film placed above. ACS Appl Mater Interfaces 2014;6:11333-40. doi:10.1021/am501870t.

[24] Yadavalli NS, Linde F, Kopyshev A, Santer S. Soft Matter Beats Hard Matter: Rupturing of Thin Metallic Films Induced by Mass Transport in Photosensitive Polymer Films. ACS Appl Mater Interfaces 2013;5:7743-7. doi:10.1021/am400682w.

[25] Lee C, Wei X, Kysar JW, Hone J. Measurement of the elastic properties and intrinsic strength of monolayer graphene. Science 2008;321:385-8. doi:10.1126/science.1157996.

[26] Di Florio G, Bründermann E, Yadavalli NS, Santer S, Havenith M. Graphene Multilayer as Nanosized Optical Strain Gauge for Polymer Surface Relief Gratings. Nano Lett 2014;14:5754-60. doi:10.1021/nl502631s.

[27] Seki T. Photoresponsive self-assembly motions in polymer thin films. Curr Opin Solid State Mater Sci 2006;10:241-8. doi:10.1016/j.cossms.2007.08.002.

[28] Uekusa T, Nagano S, Seki T. Highly Ordered In-Plane Photoalignment Attained by the Brush Architecture of Liquid Crystalline Azobenzene Polymer. Macromolecules 2009;42:312-8. doi:10.1021/ma802010x.

[29] Kopyshev A, Lomadze N, Feldmann D, Genzer J, Santer S. Making polymer brush photosensitive with azobenzene containing surfactants. Polymer 2015;79:65-72. doi:10.1016/j.polymer.2015.09.023.

[30] Igarashi K, Kashiwagi K. Modulation of cellular function by polyamines. Int J Biochem Cell Biol 2010;42:39-51. doi:10.1016/j.biocel.2009.07.009.

[31] Agostinelli E, Marques MPM, Calheiros R, Gil FPSC, Tempera G, Viceconte N, et al. Polyamines: fundamental characters in chemistry and biology. Amino Acids 2010;38:393403. doi:10.1007/s00726-009-0396-7.

[32] Pegg AE. Mammalian polyamine metabolism and function. IUBMB Life 2009;61:880-94. doi:10.1002/iub.230. 
[33] Williams K. Modulation and Block of Ion Channels: A New Biology of Polyamines. Cell Signal 1997;9:1-13. doi:10.1016/S0898-6568(96)00089-7.

[34] Gosule LC, Schellman JA. Compact form of DNA induced by spermidine. Nature 1976;259:333-5. doi:10.1038/259333a0.

[35] Chattoraj DK, Gosule LC, Schellman JA. DNA condensation with polyamines. J Mol Biol 1978;121:327-37. doi:10.1016/0022-2836(78)90367-4.

[36] Wilson RW, Bloomfield VA. Counterion-induced condensation of deoxyribonucleic acid. A light-scattering study. Biochemistry 1979;18:2192-6. doi:10.1021/bi00578a009.

[37] Takahashi M, Yoshikawa K, Vasilevskaya V V., Khokhlov AR. Discrete Coil-Globule Transition of Single Duplex DNAs Induced by Polyamines. J Phys Chem B 1997;101:9396401. doi:10.1021/jp9716391.

[38] Vijayanathan V, Thomas T, Thomas TJ. DNA Nanoparticles and Development of DNA Delivery Vehicles for Gene Therapy. Biochemistry 2002;41:14085-94. doi:10.1021/bi0203987.

[39] Venancio-Marques A, Bergen A, Rossi-Gendron C, Rudiuk S, Baigl D. Photosensitive polyamines for high-performance photocontrol of DNA higher-order structure. ACS Nano 2014;8:3654-63. doi:10.1021/nn500266b.

[40] Bain ED, Dawes K, Özçam AE, Hu X, Gorman CB, Šrogl J, et al. Surface-Initiated Polymerization by Means of Novel, Stable, Non-Ester-Based Radical Initiator. Macromolecules 2012;45:3802-15. doi:10.1021/ma300491e.

[41] Jones DM, Brown AA, Huck WTS. Surface-Initiated Polymerizations in Aqueous Media: Effect of Initiator Density. Langmuir 2002;18:1265-9. doi:10.1021/la011365f.

[42] Brown AA, Azzaroni O, Fidalgo LM, Huck WTS. Polymer brush resist for responsive wettability. Soft Matter 2009;5:2738. doi:10.1039/b902179e.

[43] Zakrevskyy Y, Cywinski P, Cywinska M, Paasche J, Lomadze N, Reich O, et al. Interaction of photosensitive surfactant with DNA and poly acrylic acid. J Chem Phys 2014;140:044907. doi:10.1063/1.4862679.

[44] Zakrevskyy Y, Richter M, Zakrevska S, Lomadze N, von Klitzing R, Santer S. LightControlled Reversible Manipulation of Microgel Particle Size Using Azobenzene-Containing Surfactant. Adv Funct Mater 2012;22:5000-9. doi:10.1002/adfm.201200617.

[45] Konradi R, Rühe J. Binding of Oppositely Charged Surfactants to Poly(methacrylic acid) Brushes. Macromolecules 2005;38:6140-51. doi:10.1021/ma047692m.

[46] Tomlinson MR, Genzer J. Evolution of surface morphologies in multivariant assemblies of surface-tethered diblock copolymers after selective solvent treatment. Langmuir 2005;21:11552-5. doi:10.1021/la051523t.

[47] Brittain WJ, Minko S. A structural definition of polymer brushes. J Polym Sci Part A Polym Chem 2007;45:3505-12. doi:10.1002/pola.22180. 